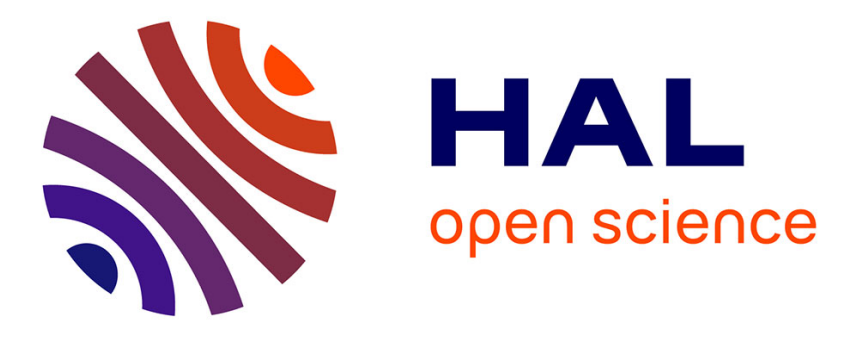

\title{
Modélisation, simulation et analyse de propriétés de réseaux orbitèles
}

\author{
Didier Josselin, Sonia Chekir, Alain Pasquet, Vincent Labatut, Yvan \\ Capowiez, Christophe Mazzia, Yezekael Hayel, Adrien Lammoglia, Cyrille \\ Genre-Grandpierre, Dieter Mitsche, et al.
}

\section{To cite this version:}

Didier Josselin, Sonia Chekir, Alain Pasquet, Vincent Labatut, Yvan Capowiez, et al.. Modélisation, simulation et analyse de propriétés de réseaux orbitèles. Revue Internationale de Géomatique, 2015, 25 (4), pp.515-536. 10.3166/RIG.25.515-536 . hal-01249881

\section{HAL Id: hal-01249881 \\ https://hal.science/hal-01249881}

Submitted on 11 Jun 2018

HAL is a multi-disciplinary open access archive for the deposit and dissemination of scientific research documents, whether they are published or not. The documents may come from teaching and research institutions in France or abroad, or from public or private research centers.
L'archive ouverte pluridisciplinaire HAL, est destinée au dépôt et à la diffusion de documents scientifiques de niveau recherche, publiés ou non, émanant des établissements d'enseignement et de recherche français ou étrangers, des laboratoires publics ou privés. 


\section{Modélisation, simulation et analyse de propriétés de réseaux orbitèles}

\author{
Didier Josselin ${ }^{1,2} \quad$ Sonia Chekir ${ }^{2}$ \\ Yvan Capowiez ${ }^{4} \quad$ Christophe Mazzia ${ }^{5}$ \\ Cyrille Genre-Grandpierre ${ }^{1}$ \\ Alain Pasquet ${ }^{3}$ \\ Yezekael Hayel ${ }^{2}$ \\ Dieter Mitsche ${ }^{7}$
}

Vincent Labatut ${ }^{2}$

Adrien Lammoglia ${ }^{6,1}$

Frédéric Patras ${ }^{7}$

Juin 2015

\section{Résumé}

Dans le cadre du projet de recherche Urbi\&Orbi, l'objectif de cet article est de proposer une méthode de construction et d'analyse des réseaux orbitèles numériques afin d'étudier certaines de leurs propriétés théoriques, avant toute application réelle en milieu urbain ou anthropisé. Les outils utilisés sont un Système d'Information Géographique associant QGIS et PostGIS et un simulateur de modèles à base d'agents (NetLogo). Ces types de réseau sont comparés aux réseaux rectilinéaires et en treillis. Une propriété dite de rectitude (ou straightness) et une mesure de centralité intermédiaire (ou «charge») du réseau sont étudiées et discutées.

Mots-clés : Orb-webs, spatial modelling, optimization, betweeness centrality, efficiency, graphs, GIS, MAS.

\section{Contexte et objectifs}

Le projet Urbi\&Orbi associe des scientifiques de différents laboratoires avignonnais, lorrain et niçois comprenant des biologistes, des géographes, des informaticiens et des mathématiciens. Au sein du champ de recherche portant sur le biomimétisme, dont l'objet est d'imiter la nature dans ses «inventions» les plus efficaces $[11,2]$, le projet Urbi\&Orbi a pour but de comparer, dans leur forme et leur usage, les structures dites «orbitèles» des toiles d'araignées [28, 29] avec les réseaux de type radioconcentrique de structures urbaines qui s'en rapprochent (i.e. possédant un centre et un mode de construction centrifuge plus ou moins monotone). II se base sur l'hypothèse que l'araignée a développé au fil du temps géologique (depuis l'ère carbonifère) des stratégies rationnelles d'exploitation optimale ( «optimal foraging») des ressources du milieu [14]. La question est de savoir si des toiles ayant une telle structure peuvent potentiellement se traduire dans des formes urbaines optimales à développer (réseaux internet, de transport, d'aduction d'eau ou d'énergie). Le projet s'inscrit dans la mouvance du biomimétisme urbain. Le biomimétisme concerne notamment le secteur industriel, l'écomimétisme, le secteur agricole. La mixité écologie-ville constitue une des formes prometteuses d'évolution des territoires urbains hybrides et durables, notamment du point de vue énergétique. Dans ce contexte, les résultats de ces travaux pourront peut-être inspirer les décideurs dans la mise en place de nouvelles politiques d'aménagement dans les territoires urbains, grâce à des métriques et des capacités renouvelées de leurs réseaux, des points de vue structurel et fonctionnel.

\footnotetext{
${ }^{1}$ UMR 7300 ESPACE, CNRS, Université d'Avignon, UNSA

${ }^{2}$ Laboratoire d'Informatique d'Avignon, Université d'Avignon

${ }^{3}$ UR AFPA - INRA, Université de Lorraine

${ }^{4}$ INRA d'Avignon

${ }^{5}$ Institut Mediterranéen de Biodiversité et d'écologie marine et continentale, UMR 7253, Université d'Avignon, AMU, CNRS, IRD

${ }^{6}$ UMR 7324 CITERES, équipe LAT

${ }^{7}$ Laboratoire de Mathématiques J.A Dieudonné, UMR CNRS-UNS N7351
} 
L'efficacité des réseaux est par exemple intéressante d'un point de vue énergétique. Ainsi, la "ville biomimétique» de Vélizy-Villacoublay développe une réflexion et des actions en termes d'urbanisme et d'optimisation des réseaux [26]. Un des enjeux soulevés par ce type d'agglomération est l'intégration intelligente des sources énergétiques à l'avenir (solaire, géothermie, etc.). Où placer les capteurs de façon optimale pour que le transport d'énergie minimise les pertes, mutualise les matériels centralisés et réduise les risques de pertes de fluide en cas de rupture d'une infrastructure? Les retombées de nos travaux peuvent ainsi concerner à terme des structures réticulaires à différentes échelles. Autre exemple: au niveau des quartiers, des réseaux de collecte des eaux usées ou d'adduction d'eau potable peuvent être (re)dessinés. On peut imaginer de nouveaux éco-quartiers dont le centre serait des lieux collectifs, collecteurs et consommateurs d'énergie (laverie, salle commune) et la périphérie accueillerait l'habitat individuel (pourvu de panneaux solaires en série), le tout organisé autour de chemins orbitèles entourant des jardins et des espaces récréatifs. À une échelle moins fine, c'est l'organisation d'une ville entière qui pourrait être dessinée de cette façon avec tous ses réseaux. Toutefois, la dépendance à l'empreinte des formes urbaines existantes laisse nettement moins de marges de manœuvre dans l'aménagement urbain de villes déjà construites. En définitive, les réseaux orbitèles peuvent avoir une utilité pour la maîtrise et l'optimisation des villes champignons à croissance rapide, telles qu'on peut les observer dans les pays émergents (Asie, Afrique et Amérique du sud).

Dans ce contexte, cet article, première étape du projet Urbi\&Orbi, a pour but de construire des réseaux orbitèles numériques afin d'étudier leurs propriétés théoriques, avant toute application éventuelle en milieu anthropisé. Les outils utilisés sont un Système d'Information Géographique et un simulateur de modèles à base d'agents. Ces types de réseaux sont comparés aux réseaux rectilinéaires et en treillis. Deux propriétés de la théorie des graphes sont étudiées et discutées: la rectitude, correspondant à une efficacité de déplacement relatif sur le réseau et la centralité intermédiaire, identifiant la charge de flux pouvant passer sur un tronçon ou un carrefour du réseau.

\section{Les réseaux orbitèles dans la Nature et la Société}

\subsection{Toiles d'araignées}

Parmi tous les animaux, les araignées se caractérisent par la production de soie qu'elles utilisent dans plusieurs de leurs contructions (cocons, abris, retraites et pièges pour la capture des proies). Parmi ces pièges utilisés par plus de la moitié des espèces, la toile géométrique est certainement la plus spectaculaire. Elle allie une redoutable efficacité grâce aux propriétés physiques de la soie lors de l'interception des proies à une importante économie d'énergie, l'ensemble ne représentant que la production de moins d'un milligramme de soie pour la construction d'une toile. Cette construction est le résultat d'un ensemble de comportements stéréotypés, mais les toiles présentent une certaine variabilité de leurs paramètres indiquant que l'araignée est capable de tenir compte d'informations extérieures pour ajuster sa construction.
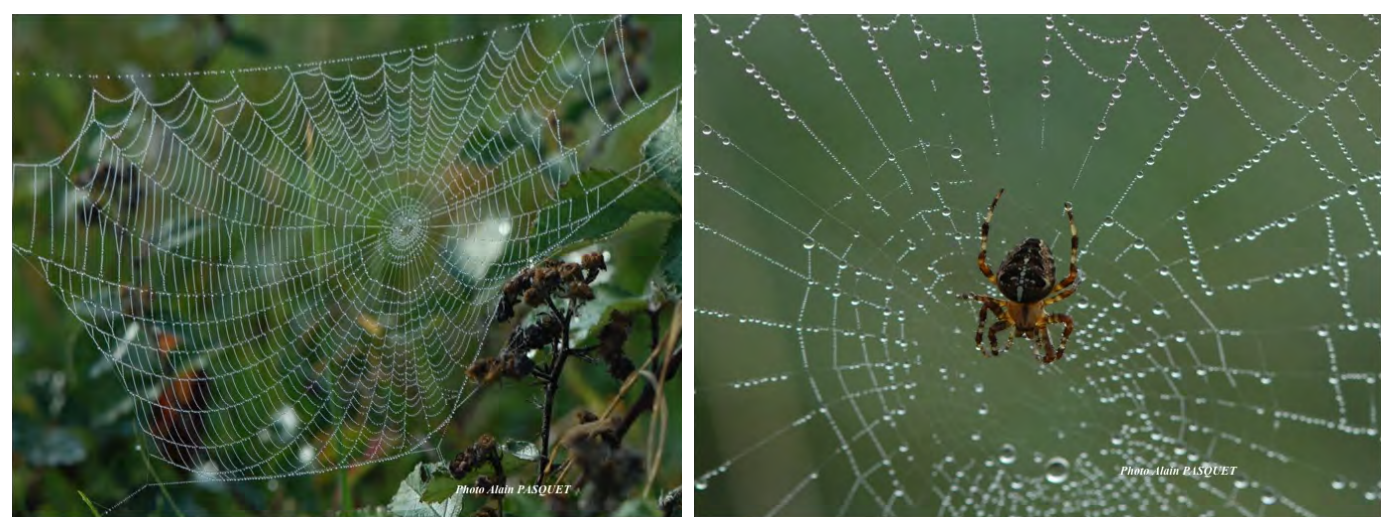

FIGURE 1 - Photographies de toiles d'araignée orbitèles, Araneus diadematus (Source : Alain Pasquet)

Dans des conditions normales, la toile géométrique se caractérise par deux critères : son 
apparente régularité et la quasi-absence de défauts (Figure 1). La régularité est liée à une série de rayons supportant une spirale dont les tours sont espacés de façon régulière, laissant apparaître un parallélisme important. Cette construction s'appuie sur une série d'étapes. L'araignée met en place sa toile en tissant d'abord un cadre qui va supporter les rayons, à partir duquel elle construit, du centre vers la périphérie, une spirale provisoire de progression logarithmique [30]. Elle va ensuite utiliser cette spirale comme guide pour tisser une spirale définitive de l'extérieur vers le centre, cette fois de progression arithmétique. Elle termine par un remodelage du centre de la toile.

L'ensemble des comportements qui sous tendent une telle construction est connu et a pu être modélisée par des algorithmes simples [15]. De plus, l'optimalité de la localisation du centre et de l'orientation de l'araignée a été démontrée $[32,18]$. Ces différents travaux étudient le modèle de toile de façon réaliste, en se calquant sur le comportement des araignées. À notre connaissance, aucune étude généralisée de la structure réticulaire orbitèle en tant que telle et de ses fonctionnalités en termes d'accessibilité n'a été réalisée, même si l'analogie informatique avec l'araignée existe dans l'analyse des réseaux [23] ou la modélisation d'araignées sociales par Système Multi-Agents [3]. À terme, la question est de savoir si cette forme géométrique possède des propriétés d'optimisation spécifiques et si celles-ci sont transposables à des réseaux anthropisés.

\subsection{Réseaux anthropiques radioconcentriques}

Avant tout, il convient de différencier, en dépit de leur ressemblance, les réseaux orbitèles des réseaux radioconcentriques. En effet, dans les réseaux orbitèles, par construction, la continuité des spires est assurée : on peut passer successivement d'un tronçon concentrique à un autre, du centre jusqu'à l'extrême périphérie, avec parfois une rupture à la séparation du moyeu (centre) et du reste de la toile. Cette propriété n'est pas valable pour les réseaux radioconcentriques où les cercles décrits ne se rejoignent pas et sont donc séparés par des tronçons de rayons. Toutefois, notons que d'une part, il existe un quasi parallélisme entre les arêtes concentriques des graphes dans les deux types de réseau, et que, d'autre part, la différence d'angle induisant cette différence est faible au regard de la structure observable dans son ensemble.

De fait, on peut déjà observer des formes de réseaux qui s'apparentent à des réseaux orbitèles dans certaines formes ou infrastructures urbaines anciennes ou actuelles (cf. Figures 2, 3 et 4), comme les charpentes de kiosques ou de clochers, les hémicycles (parlement européen de Strasbourg, théâtre antique d'Orange), voire même les vastes champs d'héliostats (Californie). II existe également aux Etats-Unis d'Amérique des villes de forme parfaitement radioconcentriques, dites villes circulaires. En Orient et dans le sud de la France, on peut aussi observer des réseaux d'irrigation de forme relativement similaire (bien qu'ayant généralement moins de cercles parallèles), correspondant à des modes d'exploitation relativement collectifs et raisonnés de l'espace agricole (exemple: l'étang de Montady dans l'Hérault).
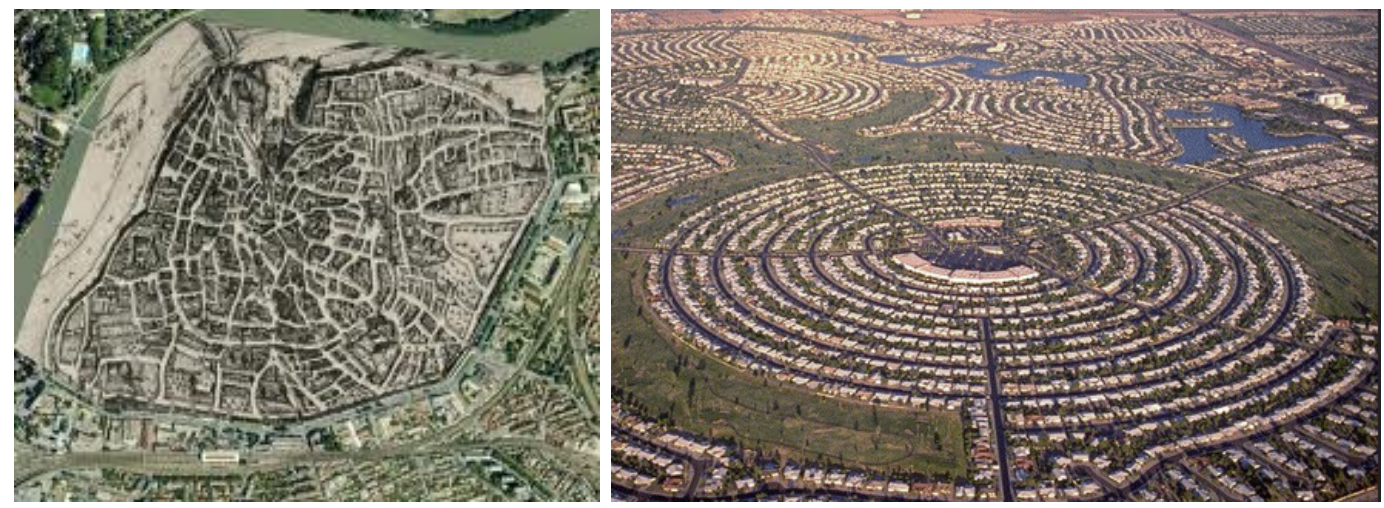

FIgURE 2 - Réseaux urbains de type radioconcentrique : ancien plan d'Avignon et ville circulaire américaine (sources: Auguste Bonnin et Google)

Une des questions traitées dans cette problématique concerne la mise en œuvre de réseaux à différentes échelles dans la ville dont l'objectif serait d'assurer un drainage et une accessibilité 
efficaces [8], tout en réduisant l'usage des matériaux de construction. Quelle que soit la forme orbitèle observée, le centre jouit d'une position privilégiée, tantôt clé de voute d'une structure porteuse, tantôt centre de gravité vu de (et voyant) tous les points du réseau ou collecteur des flux. Certaines formes positionnent également ce collecteur à l'extérieur de la toile, comme le font d'ailleurs certaines araignées. C'est donc le point de vue global de l'optimisation et de la robustesse fonctionnelles de la forme réticulaire orbitèle qui est considéré dans cette recherche.
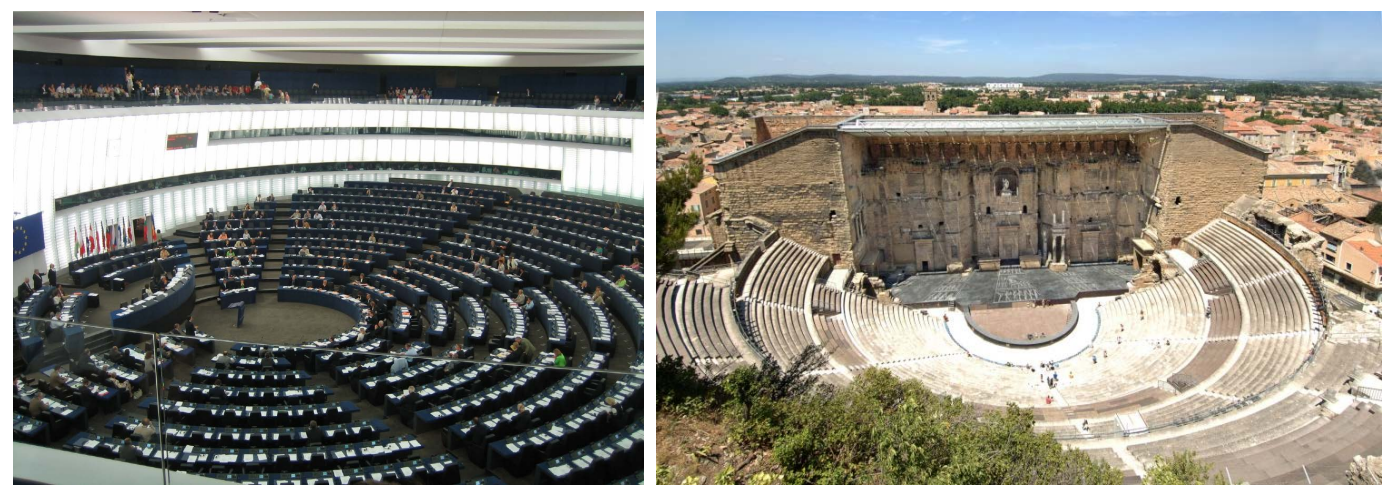

FIGURE 3 - Formes radioconcentriques de (mise en) scène : hémicycle européen et théâtre antique d'Orange (sources : Euractiv et Avignon \& Provence)

Côté économie spatiale et géographie, des travaux ont abordé cette question notamment par le biais de la métrique circum-radiale [24] qui permet d'obtenir une acessibilité parfois plus rapide en favorisant les déplacements périphériques. Les modèles radioconcentriques de Burgess (1924) ou en secteurs de Hoyt (1939) ont modélisé des structures radioconcentriques avec des cercles ou des secteurs définissant des aires ayant des fonctions urbaines particulières plus ou moins connectées au centre. La notion de centre apparaît également dans les modèles de Christaller [4] et de Lösch [17]. Dans la théorie des lieux centraux, on peut ainsi observer les aires de distribution de biens et de services sous forme d'hexagones réguliers, l'originalité étant que ces aires sont imbriquées et hiérarchisées. Par ailleurs, l'analogie apparente entre la structure des toiles et les réseaux urbains a déjà été soulignée dans la littérature $[5,20,31]$ et plusieurs métaphores utilisent la toile d'araignée, pour décrire les flux ou les chemins dans l'espace géographique [22, 25, 16], ou bien, en informatique, pour modéliser des comportements sur des réseaux [13].
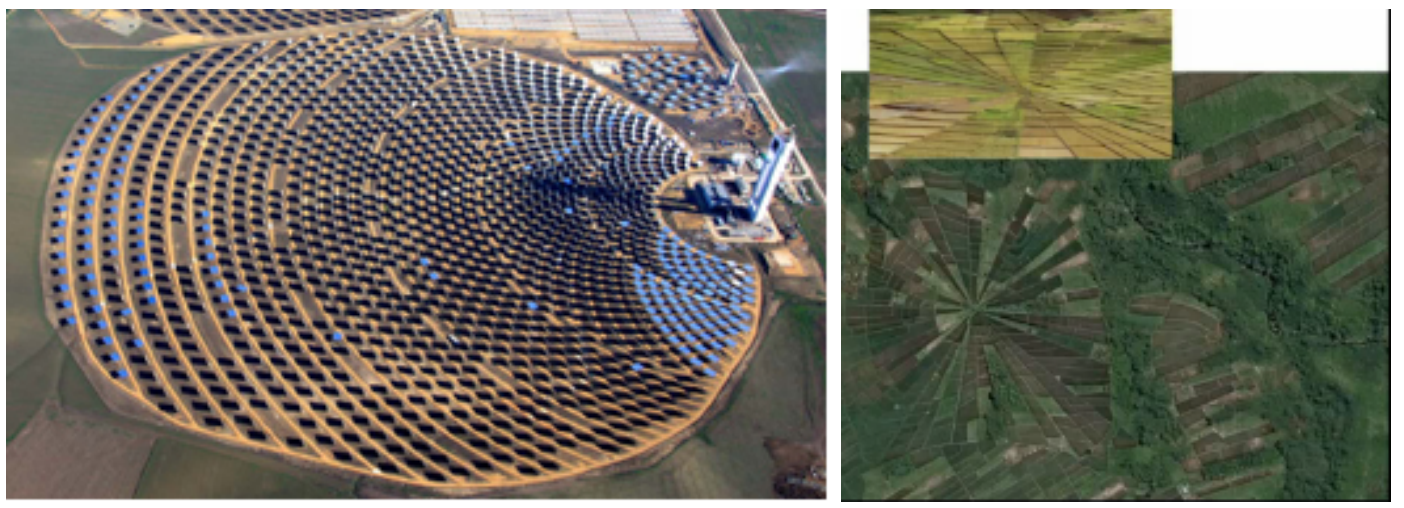

FIGURE 4 - Emprise spatiale de réseaux radioconcentriques anthropiques : champ d'héliostats aux États-Unis et réseau d'irrigation agricole au Maroc (source : Google)

Toutefois, il n'existe pas d'analyse objective décrivant et comparant quantitativement ces différents types de réseaux orbitèles, ni de réflexion sur l'apport potentiel des propriétés des formes des toiles d'araignées géométriques sur les réseaux anthropiques. De même, peu de travaux ont analysé en détails les propriétés de réseaux typiques géométriques [27] et aucun ne les a comparés aux formes orbitèles. Cet article relate la première étape de ce projet : la modélisation des toiles d'araignée orbitèles et leur comparaison à des réseaux maillés théoriques (rectilinéaires et en treillis), à l'aide de deux indices fonctionnels simples de la théorie des graphes, développés dans 
un environnement géomatique.

\section{Modélisation des réseaux orbitèles}

\subsection{Construction et fonction de la structure orbitèle}

La structure de la toile d'araignée change de façon importante dans ses trois phases de construction. La première phase consiste à construire la structure de base sur laquelle les spires de soie vont être tissées et les rayons vont s'insérer. Pour cela, l'araignée utilise la gravité. Dans une seconde phase, l'animal densifie le nombre de rayons. En partant du centre, elle construit pas à pas, dans une troisième phase, les spires de sa toile, c'est à dire les sections de soie reliant les rayons. Nous ne nous attarderons pas sur la différence de construction entre les spires du centre de la toile (plus serrées) et les spires gluantes de la partie extérieure (spirale de capture), qui couvrent la majorité de la surface. Positionnée au centre ou dans une retraite à l'extérieur de la toile, l'araignée dispose ainsi d'un accès privilégié à l'ensemble des points de la toile, en passant préférentiellement par le centre. En effet, le centre est le meilleur point de la structure pour percevoir les informations en provenance de la toile.

Nous proposons un algorithme développé en Python, qui aboutit à une structure topologique de toile orbitèle, sans toutefois suivre exactement la séquence de construction de la toile utilisée par l'araignée.

\subsection{Algorithme de construction de la toile orbitèle}

L'algorithme proposé se fonde sur la forme géomérique finale de la toile et en simplifie la construction (cf. image de droite de la Figure 6). Cherchant à obtenir une toile théorique avec des éléments géométriques comparables à ceux des réseaux maillés, nous n'appliquons pas de progression arithmétique, car celle-ci reste faible et par ailleurs sa valeur peut varier selon les espèces. La toile est ainsi composée de deux parties : le moyeu (la zone proche du centre où les spires sont plus serrées) et l'armature (la zone extérieure où les spires sont moins serrées). Entre ces deux parties, il peut y avoir un espace. La taille, le nombre de spires, la distance entre les spires et le nombre des rayons dans ces parties diffèrent selon l'espèce.

Pour mieux modéliser la toile et afin de donner à l'utilisateur plus de flexibilité pour choisir sa forme, certains paramètres d'entrée sont proposés :

- la taille de la toile $(\mathrm{t})$ : distance entre le centre et le nœud le plus lointain

- le nombre des rayons (nbR)

- le nombre de spires du moyeu (NSM)

- la distance entre deux spires successives du moyeu (DSM)

- le nombre de spires de la partie extérieure (NSE)

- la distance entre deux spires successives de la partie extérieure (DSE)

En introduisant tous ces paramètres et en considérant un repère orthonormé de centre $O$, nous avons développé un algorithme qui permet de construire un graphe de nœuds et d'arcs de forme radioconcentrique régulière en spirale. L'objectif est pour nous de conserver un modèle topologique explicite et complet afin de pouvoir exécuter des recherches de plus courts chemins sur le graphe. La construction se déroule en deux étapes inverses à celles de l'araignée : la construction du moyeu (Table 1), puis de l'armature.

À chaque étape, on commence par calculer l'angle $(\alpha)$ moyen entre les rayons :

$$
\alpha=2 \pi / n b R
$$

Ensuite, on définit les coordonnées polaires de chaque nœud (intersection entre un rayon et un élément de spire) $n(i, j)$ qui se trouve sur l'intersection de la ième spire à partir du centre et du jème rayon.

Pour les nœuds du moyeu, les coordonnées sont :

$$
\left\{\begin{array}{l}
r=i * D S M+(j+1) * D S M / n b R \\
\theta=j * \alpha
\end{array}\right.
$$

Pour les nœuds de l'armature, les coordonnées sont:

$$
\left\{\begin{array}{l}
r=t-i * D S E-j * D S E / n b R \\
\theta=j * \alpha
\end{array}\right.
$$


Après la construction de tous les nœuds du graphe, on peut dessiner les arcs du réseau en reliant deux nœuds par un segment. Pour les arcs d'une spire ou d'un rayon, on obtient respectivement :

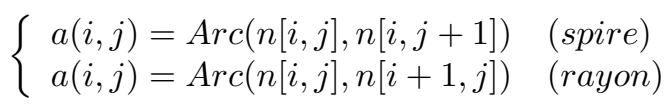

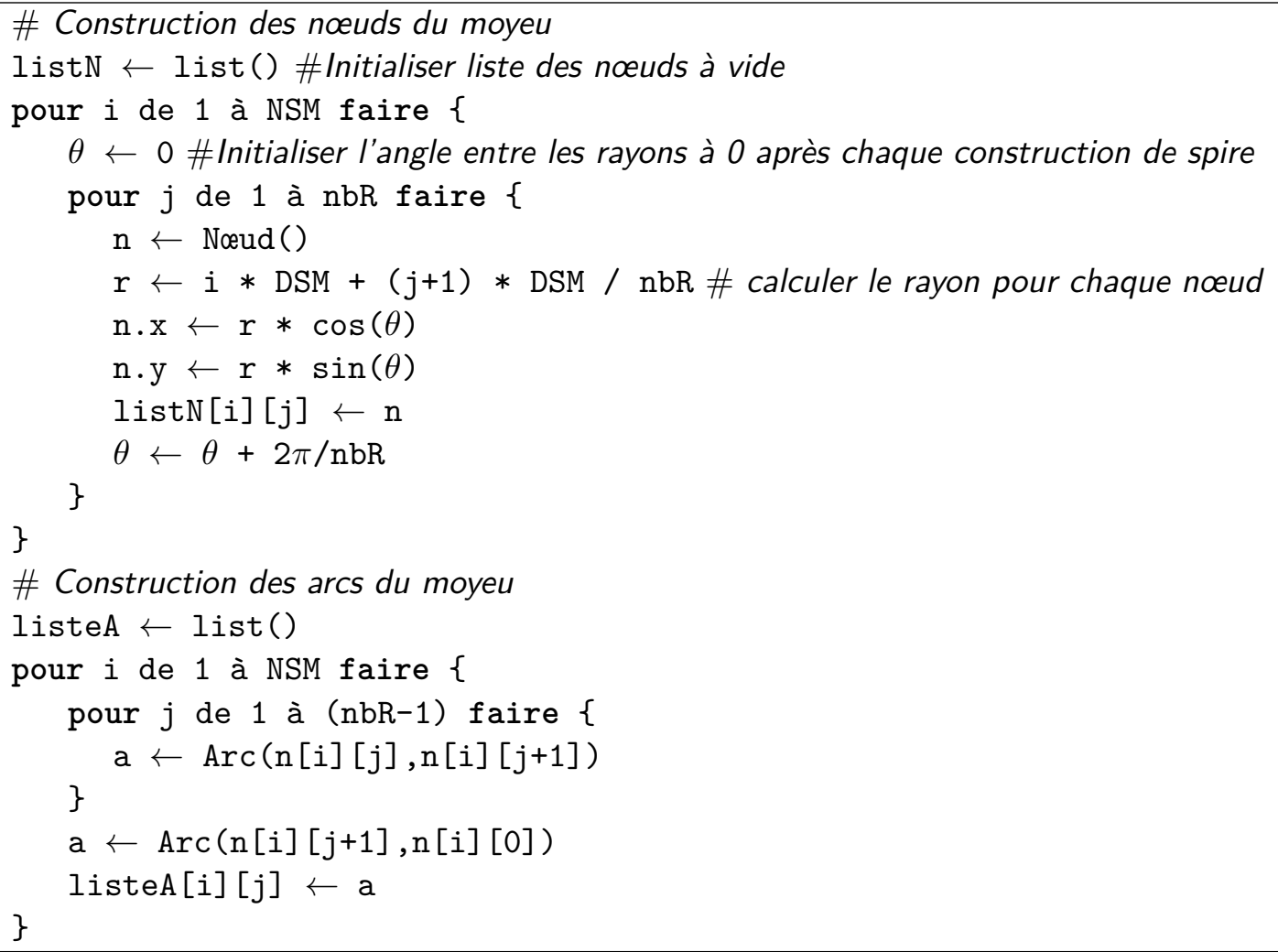

TABLE 1 - Algorithme de construction du moyeu (cf. Figure 6)

\subsection{Construction d'un réseau orbitèle au sein d'un Système d'Information Géographique}

Après le développement de l'algorithme de construction de la toile orbitèle et pour pouvoir étudier la fiabilité d'un réseau radioconcentrique, nous avons eu recours à un Système d'Informations Géographiques (Figure 5) : Quantum GIS. En plus d'être libre et d'offrir plusieurs fonctionnalités standards, ce logiciel intègre une console Python pour créer des scripts et les exécuter. Les données spatiales créées par le programme sont stockées dans le système de gestion de bases de données libre PostgreSQL. L'ajout de l'extension PostGis lui permet d'être un SGBD spatial. QGIS nous a aussi permis de créer deux fichiers SHP : I'un pour les nœuds et l'autre pour les arêtes.

Ce type de fichier SHP est exploitable par le simulateur multi-agents NetLogo grâce à l'extension GIS. Ce qui nous permet d'y intégrer le réseau pour appliquer des simulations de trafic des agents sur la structure orbitèle. Parmi les approches des systèmes complexes, nous privilégions un modèle simple en appliquant le rasoir d'Ockham (parcimonie) [1], afin d'observer l'émergence de structures récurrentes. En l'état actuel du modèle, nos agents n'intéragissent pas. Ils sont simplement réactifs [7] et simulent des déplacements rationnels indépendants (plus courts chemins). D'autres solutions techniques auraient pu être utilisées (logiciel de statistique $R$, par exemple), mais l'objectif à plus long terme est de tester la capacité de résistance des réseaux orbitèles à la montée en charge des flux en incluant les contraintes de circulation et les comportements de mobilité induits. Les Systèmes Multi-Agents paraissent donc bien adaptés. Pour le calcul des trajet des agents, nous avons utilisé pgRouting qui implémente les principaux algorithmes des plus courts chemins. En effet, ce module étend la base de données spatiales PostGIS/PostgreSQL 
pour fournir des fonctionnalités de routage, comme le calcul de plus court chemin de chaque agent avec l'algorithme de Dijkstra [6]. Grâce à son modèle entité-relation, cet environnement est adapté pour le stockage et l'analyse des données spatiales : nœuds, arêtes dont tronçons de rayons et spires, mais aussi chemins suivis par les agents.

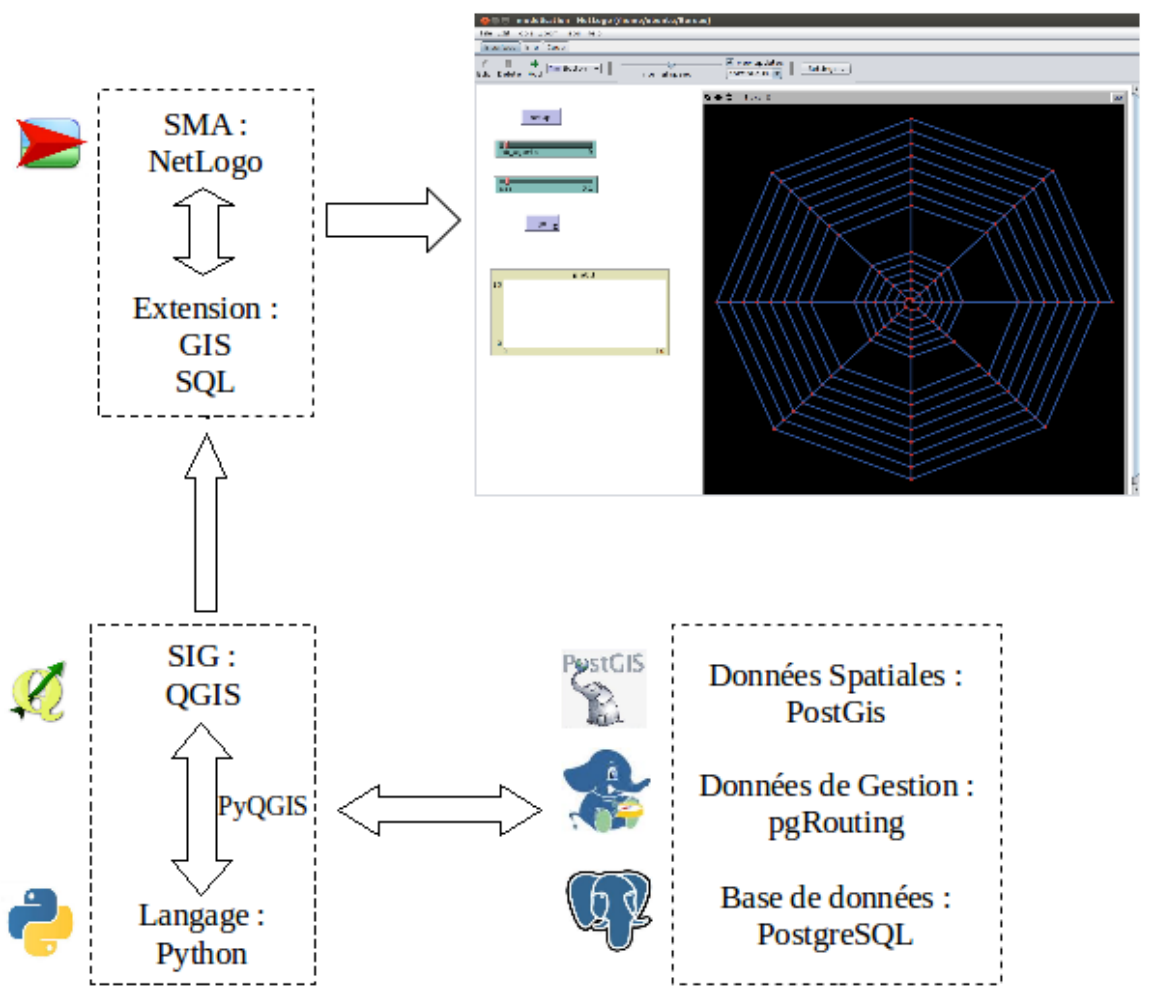

FIGURE 5 - Séquence de traitement de l'information et logiciels utilisés

\section{Analyse des propriétés des réseaux orbitèles}

\subsection{Trois types de réseaux typiques comparés}

Pour évaluer la capacité des réseaux orbitèles (Figure 6), nous les avons comparés à d'autres types de réseaux théoriques : réseaux de Manhattan et réseaux en treillis (Figure 7). Le réseau de Manhattan ou rectilinéaire, présent dans de nombreuses villes américaines, est un graphe où chaque nœud est à égale distance de ses quatre plus proches voisins. Ses arcs peuvent être soit verticaux soit horizontaux, formant des carrés de surfaces égales. Pour le réseau en treillis que nous avons modélisé, les nœuds forment un ensemble de cellules hexagonales régulières (la distance entre chaque couple de nœuds voisins est la même) et les arcs relient tous les couples de nœuds formant l'hexagone et 2 couples de nœuds opposés. Ce type de réseau en treillis se situe d'une certaine façon, de par sa forme, entre les deux autres types. II constitue un exemple parmi la grande variété des treillis (par exemple, les réseaux hexagonaux sont des formes dérivées des treillis). Les réseaux rectilinéaires et en treillis que nous avons testés sont qualifiés de réseaux «maillés» par la suite.

\subsection{Les paramètres : rectitude et centralité intermédiaire}

Pour illustrer l'idée d'accessibilité généralisée, nous utilisons un indice simple dit de rectitude (straightness en théorie des graphes), qui consiste à rapporter la distance à vol d'oiseau à la distance sur le réseau par le plus court chemin. La rectitude est une bonne estimation de l'accessibilité au moins en vitesse relative. Cela correspond à l'inverse de la circuité (aussi appelée tortuosité ou dimension d'étalement). Ce type d'indice est notamment utilisé pour évaluer la fractalité des formes côtières [19]. Lorsque la distance euclidienne est rapportée au coût en temps 

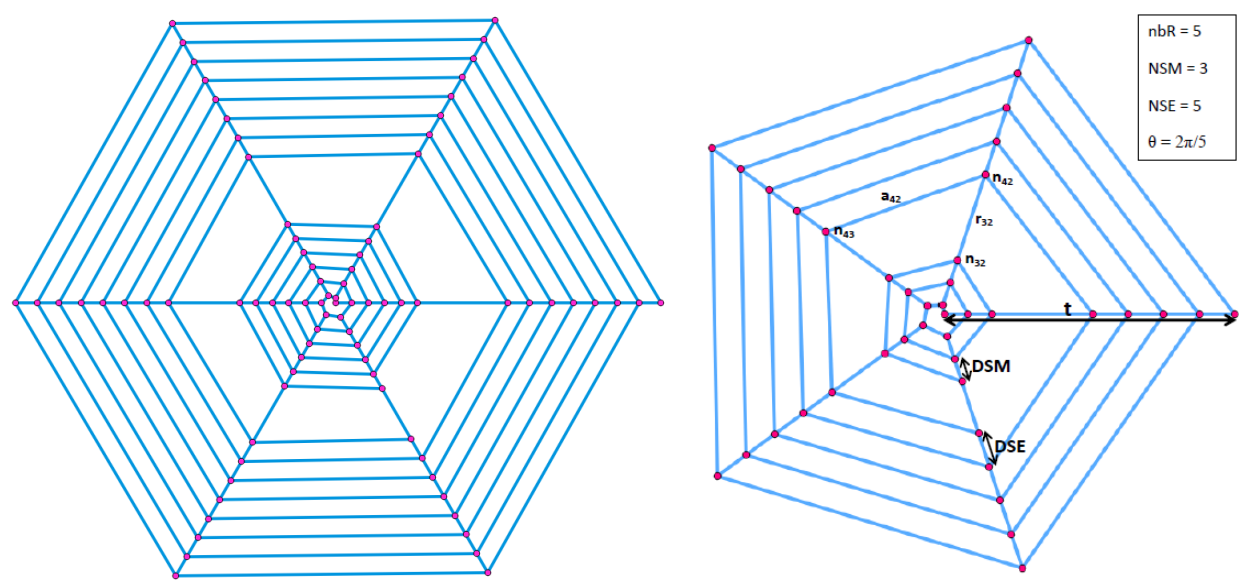

FiguRE 6 - Exemples de toiles orbitèles simulées
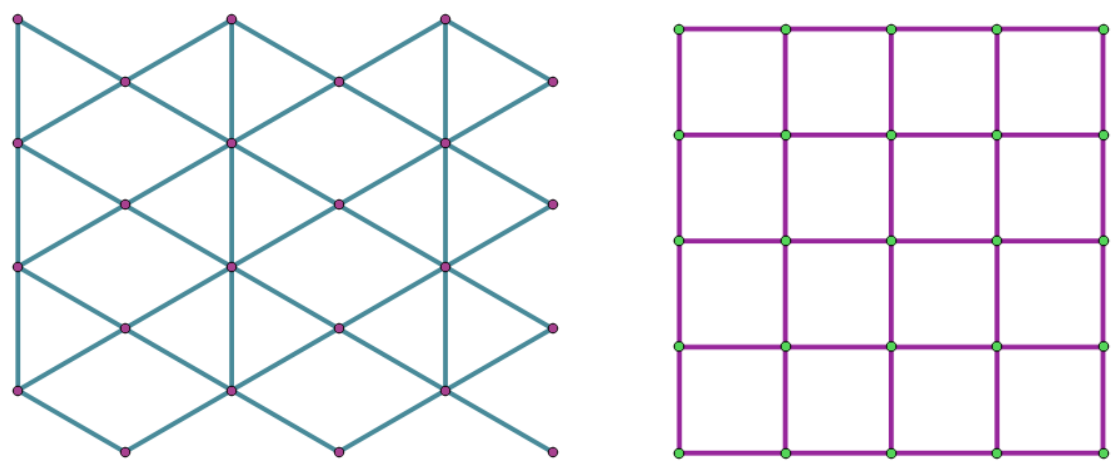

FIGURE 7 - Réseaux en treillis et de Manhattan (rectilinéaire)

sur le réseau, on parle plutôt d'efficacité [12], c'est alors une vitesse relative. Dans notre cas, la vitesse autorisée sur le réseau est constante et donc le temps de parcours est proportionnel à la distance, puisque nous n'intégrons pas la congestion éventuelle. Ainsi, lorsque la rectitude tend vers 1 , cela signifie que le trajet est direct; plus sa valeur est faible, plus les détours sont longs sur le réseau. Un exemple de détour sur une toile orbitèle est donné dans la partie gauche de la Figure 10.

La rectitude $R$ ou straightness est donc le rapport entre la distance spatiale à vol d'oiseau $d_{1}$ et la distance spatiale $d_{2}$ obtenue en suivant le plus court chemin sur le réseau (cf. Figure 8) :

$$
R=\frac{d_{1}}{d_{2}}
$$

À cet indice, traité au niveau de l'ensemble des agents, nous ajoutons la charge, mesure équivalente à la centralité intermédiaire $[9,21]$ en théorie des graphes. Dans notre cas, elle est spatiale (et pas seulement topologique) car nous considérons les distances entre les noeuds. Appliquée à des réseaux viaires [10], la centralité intermédiaire est estimée par le calcul du nombre de passages d'agents sur chaque tronçon de route. Ces agents ont des Origines et des Destinations aléatoires, mais leur trajet suit systématiquement un plus court chemin. Sur un réseau homogène fortement connecté, plus un tronçon ou une intersection de route se trouve au centre, plus sa charge est potentiellement importante, puisque la probabilité de la traverser augmente mécaniquement. Souhaitant tester la capacité des tronçons du réseau à recevoir les flux, nous localisons nos agents aléatoirement sur les nœuds du réseau et leur fixons une destination également aléatoire. Avec un nombre suffisant d'agents, on couvre peu ou prou la surface du réseau par une multitude d'origines destinations associées à leurs plus courts chemins. À chaque simulation, nous collectons la rectitude de chaque trajet d'agent et la charge pour chaque tronçon, que nous pouvons ainsi cartographier. 


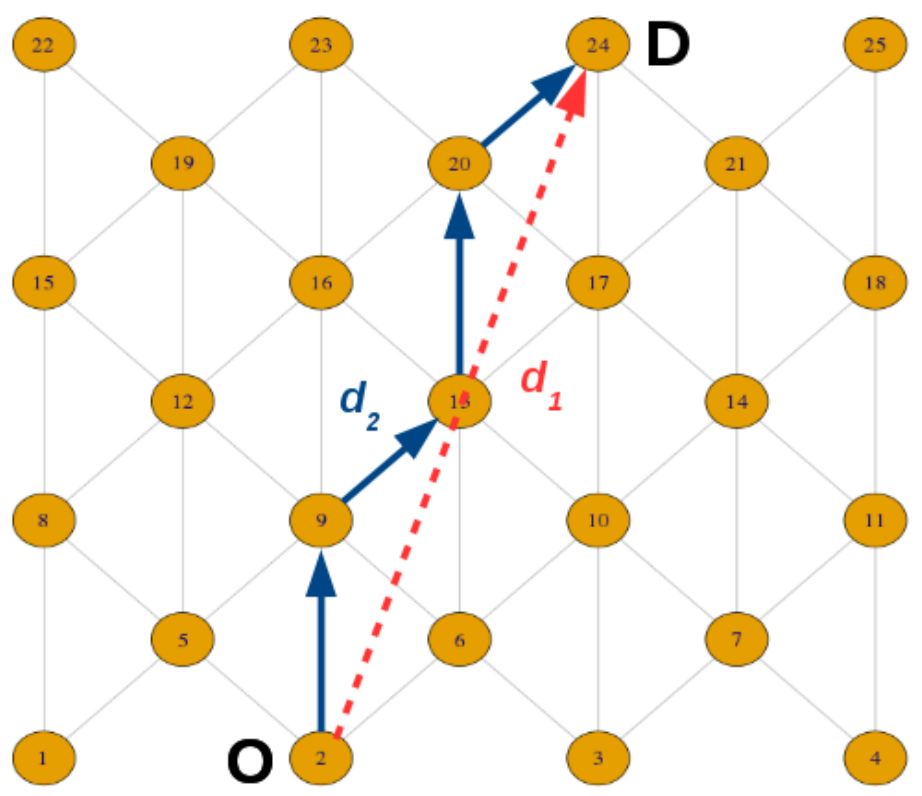

FiguRE 8 - Calcul de la rectitude entre l'origine $O$ et la destination $D$ sur un réseau en treillis : $d_{1}$ est la distance à vol d'oiseau, $d_{2}$ représente le plus court chemin sur le réseau passant par les noeuds $2,9,13,20$ et 24 .

\subsection{Modélisation à base d'agents}

Au sein du simulateur multi-agents NetLogo, nous modélisons le déplacement des agents sur les arcs, d'un nœud de départ à un nœud d'arrivé en choisissant le plus court chemin (cf. diagramme d'activité de la Figure 9). À noter que nous déplaçons chaque agent d'une très petite distance sur le réseau, ce qui permet aux agents de progresser sur le réseau, en fonction de leur position, de leur direction, de leur éventuelle rencontre d'une intersection à un nœud, mais également en fonction du plus court chemin qui leur a été assigné par pgRouting. Lors de ce processus, nous calculons continuellement le nombre de passages sur chaque arc (charge) et, à chaque fin de parcours d'agent, nous calculons la rectitude de déplacement. La Figure 10 montre un exemple de réseau théorique épuré (peu de rayons et peu de spires) dont les arcs sont affectés des comptages des agents en cours de simulation, ainsi que quelques plus courts chemins possibles. À noter qu'il peut exister fréquemment différents chemins équivalents en distance.

Pour étudier l'architecture des réseaux orbitèles, nous avons fait des simulations avec 1500 agents sur 3 modèles dont les deux premiers sont proches des toiles d'araignée observées (épeires), le troisième étant purement théorique :

- Orbitèle 1 , composé de 20 rayons et 25 spires, dont 5 spires de moyeu;

- Orbitèle 2, composé de 40 rayons et 60 spires, dont 10 spires de moyeu;

- Orbitèle 3, composé de 8 rayons et 60 spires, dont 10 spires de moyeu.

Ces modèles sont comparés aux deux autres réseaux : Manhattan et en treillis, tout en essayant d'avoir un nombre comparable d'arêtes et de nœuds (ce qui ne peut être exact, vu les différences topologiques des réseaux).

La Table 2 décrit le nombre de nœuds et d'arcs de chaque type de réseau, pour chaque modèle. Les réseaux orbitèles 1 et 3 , de Manhattan et en treillis ont des tailles à peu près similaires morphologiquement et topologiquement.

\begin{tabular}{|c|c|c|c|c|c|}
\hline & Orbitèle 1 & Orbitèle 2 & Orbitèle 3 & Manhattan & Treillis \\
\hline Nombre de nœuds & 500 & 2400 & 480 & 505 & 418 \\
\hline Nombre d'arêtes & 978 & 4758 & 950 & 966 & 975 \\
\hline
\end{tabular}

TABLE 2 - Caractéristiques des réseaux construits 


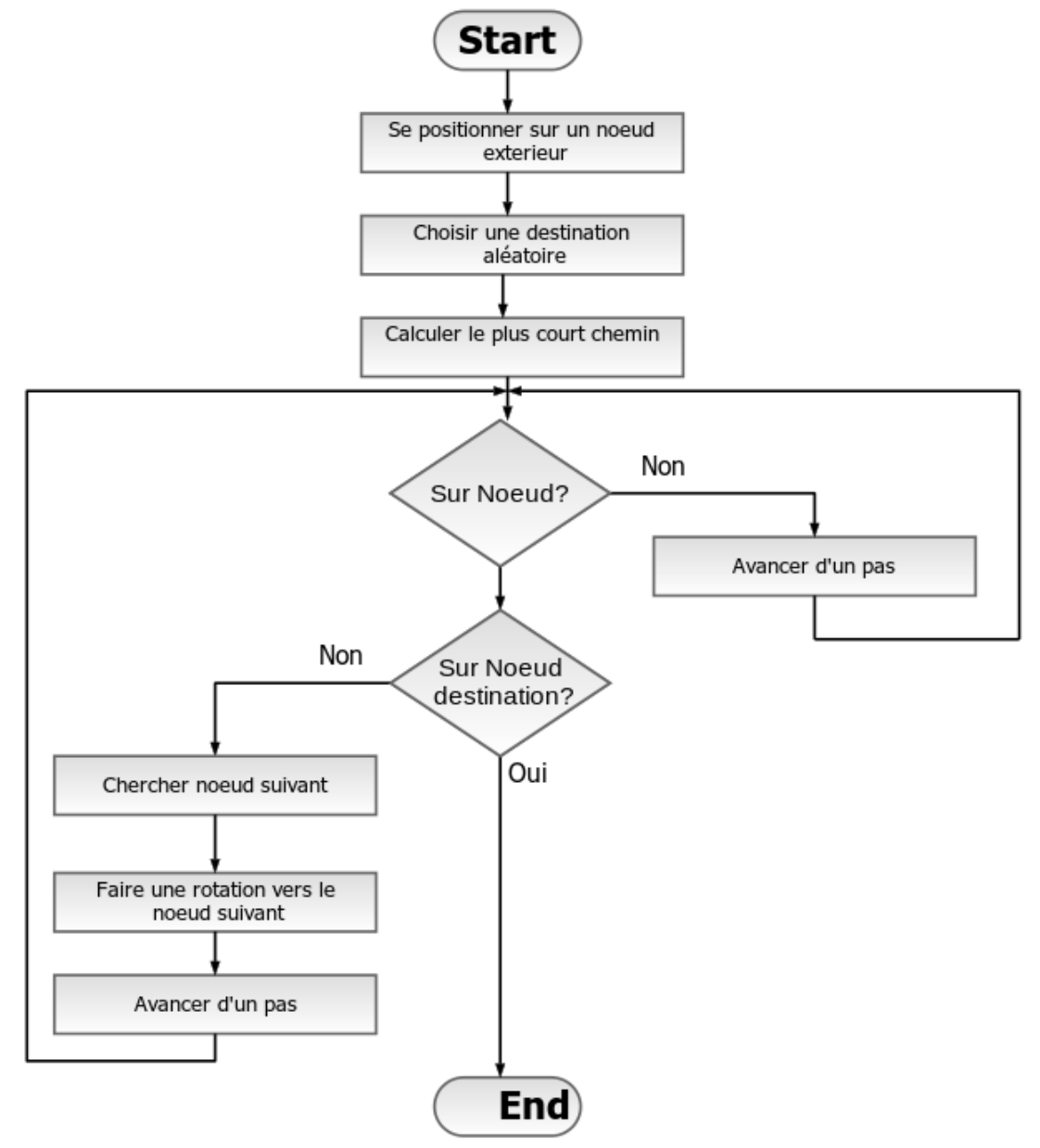

FiguRE 9 - Diagramme d'activité de déplacement des agents

\section{Résultats et discussion des simulations}

\subsection{Les réseaux orbitèles sont les plus efficaces (en termes de rectitude) pour des déplacements multi-directionnels}

Observons les résultats obtenus pour la rectitude des déplacements des agents sur le réseau. Pour ce qui concerne plus particulièrement les toiles orbitèles (Figures 11 et 12), en dépit du fait que le nombre d'arcs des rayons est à peu près égal au nombre d'arcs des spires, on observe que, même dans une configuration d'origines et de destinations aléatoires, les trajets directs (de rectitude 1) sont finalement peu nombreux en proportion, beaucoup de déplacements nécessitant des petits détours. Pour ces trois réseaux, la médiane et la moyenne des rectitudes oscillent entre 0,92 et 0,94 avec un écart-type faible de 0,06.

On observe par ailleurs deux groupes distincts : les toiles orbitèles et les réseaux de mailles régulières (Figure 13), dont les formes des histogrammes s'opposent. Pour les formes orbitèles, on note deux pics de rectitude proches de 1 et (de taille moindre) vers 0,85 . Pour les réseaux maillés, on constate le pic le plus important vers 0,7 et un pic plus petit vers 1 . Les trajets sont globalement moins directs que ceux des toiles orbitèles, même si les minima de rectitude de ces derniers peuvent être plus faibles. Cela est confirmé par les moyennes et médianes des réseaux de Manhattan et en treillis, respectivement $(0,9$ et 0,9$)$ et $(0,76$ et 0,8$)$. Les écarttypes correspondants restent faibles $(0,05$ pour le treillis et 0,1 pour le réseau rectilinéaire). Les réseaux orbitèles et rectilinéaires sont donc assez proches en termes de rectitude (caractère direct des trajets), même si les réseaux orbitèles semblent légèrement meilleurs. Cette dernière propriété reste cependant à démontrer mathématiquement (par géométrie) et son étude doit être généralisée par la simulation des trajets possibles de manière exhaustive sur ce type de réseau 

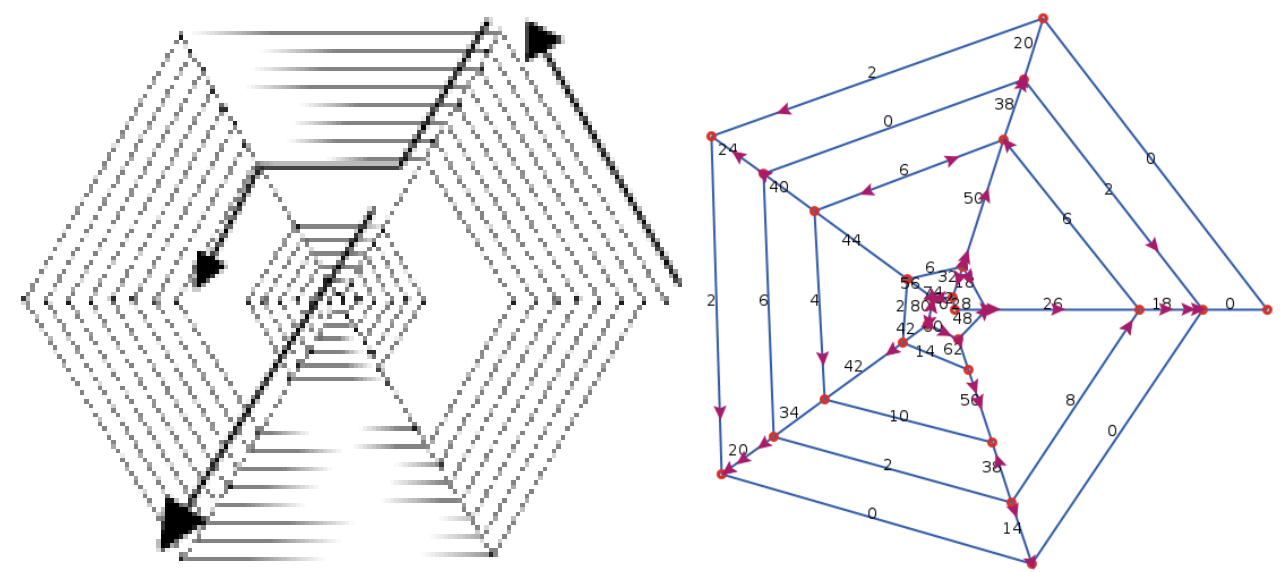

FIGURE 10 - Exemples de plus courts chemins et de calcul de charge (centralité intermédiaire) au tronçon sur des réseaux orbitèles; les chiffres indiquent le nombre de passages d'agents
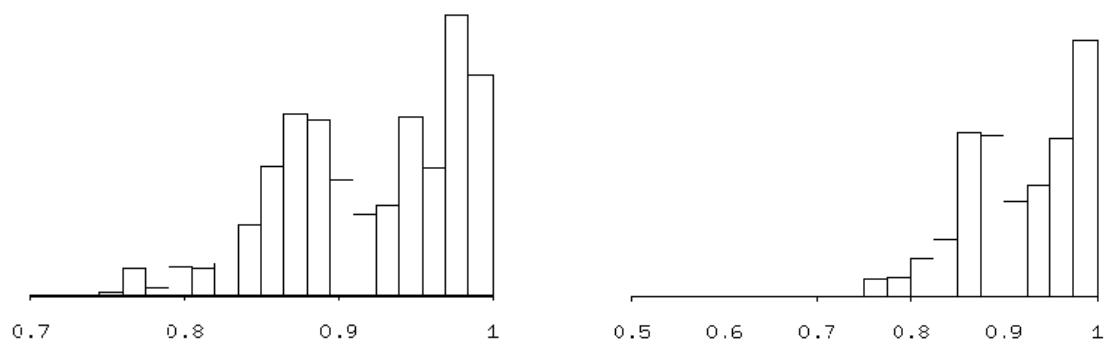

FIGURE 11 - Rectitude des trajets des agents pour 2 simulations du même réseau orbitèle 1

orbitèle. Ce travail est en cours de réalisation par l'équipe inter-disciplinaire du projet Urbi\&Orbi.

\subsection{La forme orbitèle concentre les flux sur le centre et les rayons}

Les premiers résultats portent sur la centralité intermédiaire (charge) que nous cartographions par tronçon, pour 4 des 5 réseaux étudiés. Pour les réseaux maillés, on observe une valeur progressivement plus élevée de la charge (passage des agents) de la périphérie vers le centre, qui est due à la probabilité de l'arc d'être d'autant plus traversé que sa position est centrale dans la topologie du graphe (Figure 14). Le dégradé de gris qui en résulte n'est toutefois pas parfaitement lissé, car le calcul des plus courts chemins a tendance à favoriser certains trajets par rapport à d'autres qui seraient strictement équivalents. Pour bien faire, il serait nécessaire d'introduire un choix aléatoire dans l'ensemble des chemins équivalents en distance (travaux en
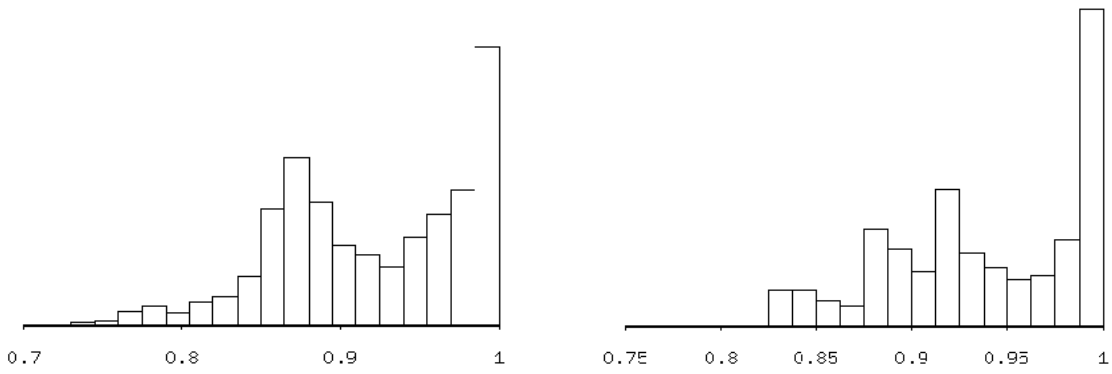

FIGURE 12 - Rectitude des trajets des agents pour les réseaux orbitèles 2 et 3 

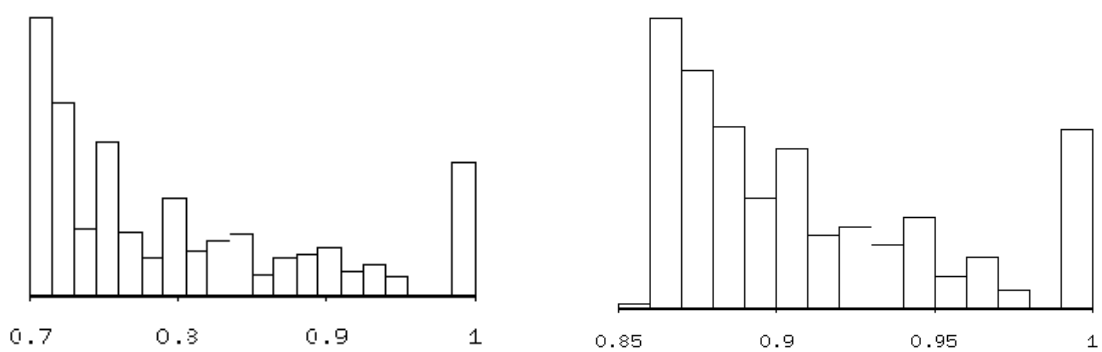

FIGURE 13 - Rectitude des trajets des agents pour les réseaux rectilinéaire (à gauche) et en treillis (à droite)
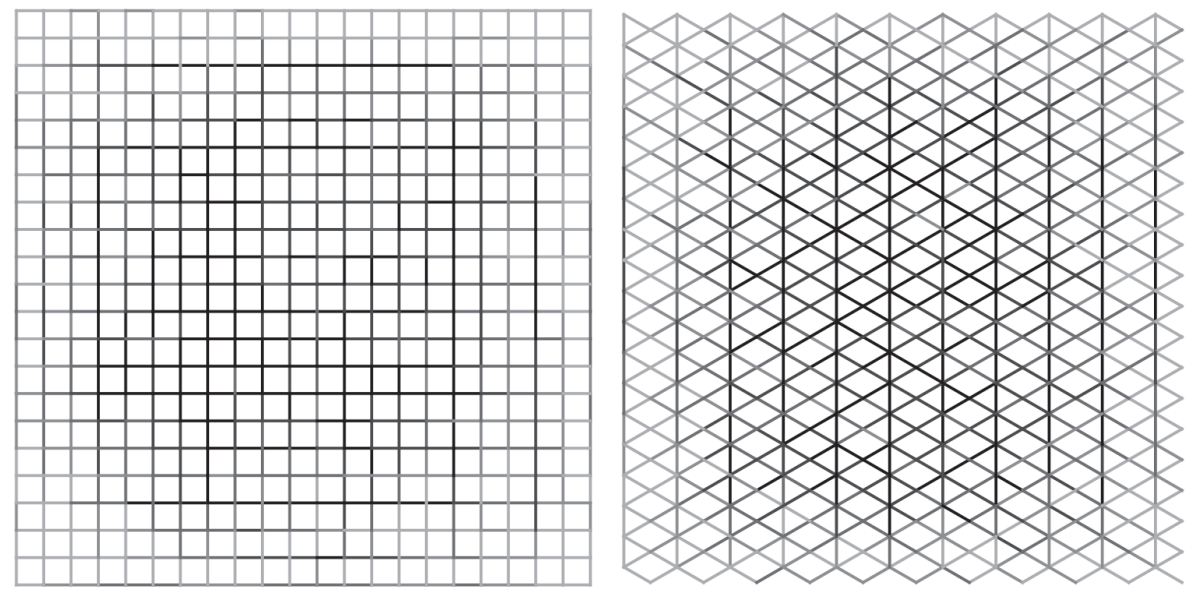

FIGURE 14 - Centralité intermédiaire (charge) des arcs sur les réseaux rectilinéaires (gauche) et en treillis (droite) ; plus le trait est foncé, plus le passage des agents a été important; un trait gris clair correspond à quelques unités de centralité intermédiaire; une couleur noire correspond à une centaine de passages d'agents.

cours).

La forme étoilée qui émerge des réseaux orbitèles est intéressante (Figure 15). D'une part, le moyeu est saturé en passage d'agents. D'autre part, selon la règle évoquée ci-dessus, les spires positionnées au même niveau que les tronçons de rayons adjacents pourraient avoir des charges équivalentes, ce qui n'est pas le cas. On constate en effet que les rayons ont une fonction de pénétration, due à leur direction centripète et leur raccordement au centre au seul nœud de degré supérieur à 4 (égal au nombre de rayons). La probabilité de passer sur les rayons est donc beaucoup plus élevée que celle des spires (d'un facteur 5 à 10 selon l'éloignement au centre). N'étant pourtant pas plus nombreux que les spires et possédant des nœuds de même degré sur le graphe (excepté au centre), les rayons concentrent, en proportion, beaucoup plus fortement les flux. Cette caractéristique forte des réseaux orbitèles, très proche du fonctionnement des réseaux urbains radioconcentriques, pourrait peut-être, à terme, être transposée sur des réseaux anthropisés dont on pourrait estimer ainsi a priori la capacité à recevoir et diffuser les flux. Si l'on ajoute une composante scalaire, on pourrait imaginer des structures composées de hubs imbriqués facilitant la diffusion des échanges, un peu comme Christaller l'avait imaginé. Toutefois, les problèmes induits de congestion et la prise en compte du temps comme coût de déplacement dans les pratiques effectives de mobilité devront être reconsidérés.

\section{Conclusion}

Nous proposons dans cet article une base méthodologique de modélisation et de construction des réseaux géométriques à partir de PostGIS, QGIS et NetLogo. Une première comparaison de deux propriétés (indice de rectitude et centralité intermédiaire) des réseaux semble montrer 

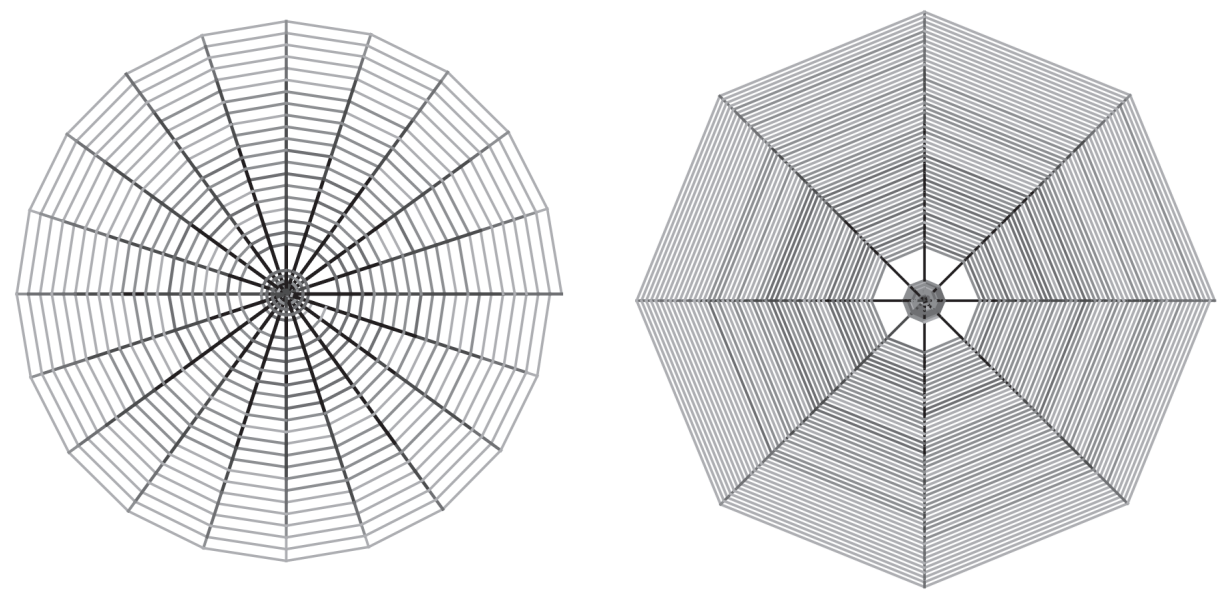

FIGURE 15 - Centralité intermédiaire (charge) des arcs sur les réseaux orbitèles 1 et 3 ; plus le trait est foncé, plus le passage des agents a été important; un trait gris clair correspond à quelques unités de centralité intermédiaire; une couleur noire correspond à une centaine de passages d'agents.

la meilleure capacité d'accessibilité sur la surface par des réseaux orbitèles face aux réseaux de Manhattan ou en treillis. En dépit de sa structure en étoile, la toile d'araignée orbitèle favorise les déplacements centre-périphérie dans une certaine mesure. Ainsi, le positionnement de la toile orbitèle pour ce qui est de l'étalement urbain et de la congestion des voies urbaines pénétrantes pose question et reste à étudier avec précision selon les types de flux observés. D'autres indicateurs doivent être ajoutés pour évaluer la capacité de ces réseaux à diffuser les flux sur la surface couverte par la toile : longueur d'arêtes, taux de couverture, robustesse des réseaux, etc. Une comparaison affinée entre les toiles orbitèles et les réseaux radioconcentriques sera également menée pour évaluer ce que la continuité de la spire peut apporter au réseau, au delà de la logique de construction de la toile.

En prolongement de ces modèles, nous envisageons d'intégrer rapidement des réseaux urbains réels (évoqués en introduction) afin d'y réaliser des calculs similaires et avec d'autres indices, pour, d'une part, positionner les réseaux viaires existant par rapport aux réseaux géométriques, d'autre part, évaluer à terme l'efficacité des réseaux orbitèles en termes d'accessibilité, objectif final du projet Urbi\&Orbi. De plus, des toiles d'araignées réelles, issues de numérisation de photographies, complèteront les supports de simulation. En lien avec les écologues, les formes et les constructions des toiles seront affinées selon les conditions locales et les espèces d'araignées, dont le comportement sera étudié en lien avec les variations des formes orbitèles. Enfin, le modèle sera prochainement amélioré en intégrant la congestion due aux co-présences d'agents et leur comportement adaptatif dans de telles situations, afin d'évaluer l'impact de telles situations sur l'équilibre des flux pour différents types de réseaux.

\section{Remerciements}

Nous remercions le Laboratoire d'Informatique d'Avignon (projet VPTT) d'avoir financé le stage de fin d'étude d'ingénierie informatique de Sonia Chekir, I'Université d'Avignon (projet d'excellence Urbi\&Orbi) et le CNRS (PEPS MOMIS) pour leur soutien à ce projet interdisciplinaire. Un grand merci également aux relecteurs de l'article de SAGEO'2014 et de la Revue Internationale de Géomatique pour leurs critiques constructives et leurs conseils avisés.

\section{Références}

[1] F. Amblard and D. Phan. Modélisation et simulation multi-agents : applications pour les Sciences de l'Homme et de la Société. Hermes Science, 2006.

[2] Janine Benyus M. Biomimétisme. Quand la nature inspire des innovations durables. Edition française de 2008, Editions Rue de l'Echiquer, Paris, 2011. 
[3] Christine Bourjot, Vincent Chevrier, and Vincent Thomas. How Social Spider Inspired An Approach To Region Detection. In First International Joint Conference on Autonomous Agents and Multiagent Systems - AAMAS 2002, page 8, Bologna, Italy, 2002.

[4] Walter Christaller. Die zentralen Orte in Süddeutschland. Eine ökonomisch-geographische Untersuchung über die Gesetzmäßigkeit der Verbreitung und Entwicklung der Siedlungen mit städtischen Funktionen. PhD thesis, Jena, G. Fischer Verlag, 1933.

[5] Thomson D'Arcy Wentworth. On growth and form. Cambridge University Press, The MacMillan Company, New-York, 1945.

[6] Edsger W Dijkstra. A short introduction to the art of programming, EWD316, 100 pages, 1971.

[7] Jacques Ferber. Les systèmes multi-agents : vers une intelligence collective. Inter Editions, Paris, 1995.

[8] Jean-Christophe Foltête, Cyrille Genre-Grandpierre, and Didier Josselin. Impacts of Road Networks on Urban Mobility, in Modeling Urban Dynamics, pages 103-128. ISTE Ltd and John Wiley \& Sons, NY, 2010.

[9] Linton Freeman. A set of measures of centrality based on betweenness. Sociometry, 40(1) : 35-41, 1977.

[10] Cyrille Genre-Grandpierre. Forme et fonctionnement des réseaux de transport : approche fractale et réflexions sur l'aménagement des villes. PhD thesis, Université de Franche-Comté, Thèse de Doctorat., 2000.

[11] Agnès Guillot and Jean-Arcady Meyer. La bionique. Quand la science imite la nature. Dunod, UniverScience, Paris, 2008.

[12] J. Gutiérrez, A. Monzòn, and J.M. Pinero. Accessibility, network efficiency and transport infrastructure planning. Environment and Planning A, 30 :1337-1350, 1998.

[13] F.Y. Huang and D.B. Skillicorn. The spider model of agents. In Samuel Pierre and Glitho Roch, editors, Mobile Agents for Telecommunication Applications, pages 209-218. Third International Workshop, MATA, Montreal, Canada, August 14-16, 2001, 2001.

[14] J.R. Krebs and N.B Davies. Behavioural Ecology : an evolutionary approach. Cambridge Mass, Blackwell Science., 1993.

[15] Thiemo Krink and Fritz Wollrath. Analysing spider web-building behaviour with rule-based simulations and genetic aclgorithms. Journal of theoretical biology, 185 :321-331, 1997.

[16] Ting Li, Diange Yang, Hua Geng, Wang Liu, Tao Zhang, and Xiaomin Lian. Spider-web road network model for in-vehicle navigation digital maps. Journal of Wuhan University of Technology (Transportation Science and Engineering), 34 :439-442, 2010.

[17] August Lösch. Die räumliche Ordnung der Wirtschaft. Eine Untersuchung über Standort, Wirtschaftsgebiete und internationalen Handel. Jena, G. Fischer Verlag, 1940.

[18] Wes Maciejewski. An analysis of the orientation of an orb-web spider. Journal of theoretical biology, 265 :604-608, 2010.

[19] Benoît Mandelbrot. Les objets fractals. Forme, hasard et dimension. Champs Sciences, 1995.

[20] Stephen Marshall. Street patterns. Spon Press, London, New-York, 2005.

[21] Eric Mermet. Conception de méthodes et d'outils d'analyse spatiale pour l'organisation, l'exploration et la représentation d'informations géographiques sur un réseau de transport. PhD thesis, Université de Marne la Vallée, COGIT, 2011. 
[22] Joao Mourinho, Teresa Galvao, and Joao Falcao e Cunha. Spider maps for location-based services improvement. In Mehdi Snene, Jolita Ralyté, and Jean-Henry Morin, editors, Lecture Notes in Business Information Processing, in Exploring Services Science, Geneva, Switzerland, 2011. Second International Conference, IESS 2011, February 16-18, 2011, SpringerVerlag.

[23] Richard Moussa, Marie Beuron-Aimar, and Pascal Desbarats. Image processing on MR brain images using social spiders, chapter 18, pages 364-382. Intech, 2011.

[24] J. Perreur and J. Thisse. Central metrics and optimal location. Journal of Regional Science, 4(3) :411-421, 1974.

[25] Zhang Qiaoping and Isabelle Couloigner. Automatic road change detection and gis updating from high spatial remotely-sensed imagery. Geo-Spatial Information Science (Quaterly), $7(2): 89-95,2004$.

[26] SOREPA. PLU de vélizy-villacoublay, révision du plan local d'urbanisme, rapport de présentation, arrêté de projet. Technical report, Commune de Vélizy-Villacoublay, 2005.

[27] Isabelle Thomas. Transportation Network and the Optimal Location of Human Activities. A numerical geography approach. Cheltenham, Edward Elgar Publishing Itd, 2002.

[28] C. Toscani, R. Leborgne, and Pasquet A. Behavioural analysis of web building anomalies in the orb-weaving spider zygiella $\mathrm{x}$-notata (araneae, araneidae). Arachnologische Mitteilungen, $43: 6-9,2012$.

[29] S. Venner, I. Chadès, M.C. Bel-Venner, A. Pasquet, F. Charpillet, and R. Leborgne. Dynamic optimization over infinite-time horizon : web-building strategy in an orb-weaving spider as a case study. Journal of Theoretical Biology, 241(4) :725-733, 2006.

[30] F. Vollrath and W. Mohren. Spiral geometry in the garden spider's orb web. Naturwissenschaften, 72 :666-667, 1985.

[31] David Wise H. Spiders in ecological webs. Cambridge University Press, 1995.

[32] Samuel Zschokke and Kensuke Nakata. Spider orientation and hub position in orb webs. Naturwissenschaften, $97: 43-52,2010$. 